Verhandlungen des Vereins zur Beförderung des Gẹ̛erbfleisses in Preussen. VIrr. Heft. Chemical News. No. 934.

Revue scientifique. No. 16, 17.

Durch Kauf:

Comptes rendus. T. LXXXV, No. 15-17.

Dingler's polytechnisches Journal. Bd. 226, Heft 1, 2.

Der Schriftführer i. V.:

E. Salkowski.
Der Vorsitzende:

C. Liebermann.

\title{
Mittheilungen.
}

442. C. Rammelsberg: Ueber das Atomgewicht des Molybdäns und die phosphormolybdänsauren Balze.

(Vorgetragen in d. Sitzung rom Verfasser.)

Das A tomgewicht des Molybdäns.

Berzeliug' Versuche vom Jahre 1818 gaben, mit dem jetzt geltenden Atomgewicht ron $\mathrm{N}$ und $\mathrm{Pb}$ berechnet, $\mathrm{Mo}=95.3$.

$\mathrm{S}$ anberg und Struve erhielten durch Verwandlung von $\mathrm{MoS}_{2}$ in $\mathrm{MoO}_{3}$ im Mittel die $\mathrm{Zahl} \mathrm{92.14;} \mathrm{allein} \mathrm{da} \mathrm{die} \mathrm{Grenzwerthe} \mathrm{von}$ 10 Versuchen 97.1 und 90.5 sind, so hat schou Strecker mit vollem Recht bemerkt, dass die Zahl 92 kein grosses Vertrauen verdiene. Später hat Dumas angekündigt, Mo sei $=96$, ohne jedoch factische Beweise mitzutheilen. Debray führte hierauf an, er habe sowohl durch Reduction der Süure in Wasserstoff; als anch durch die Analyse des Silbersalzes die Zahl 96 erhalten, jedoch auch Debray hat die Belege dafür nicht publicirt. Zuletzt hat auch Meyer Mo $=95.8$ gefanden.

Somit scheint die Zahl 96, der die alte Bestimmung Berzelius' schon nahe kam, der Wahrheit besser zu entsprechen, als die vielfach benutzte 92. Für die nachfolgenden Versuche über die Phosphomolybdate war es jedoch wünschengwerth, das Atomgewicht des Mo mittelst eigener Versuche zu bestimmen. Durch Reduction der Süure in Wasserstoff zu Metall habe ich nun gleichfalls Mo $=96.18$ erhalten und werde desshalb weiterhin die Zabl 96 benutzen.

\section{Phosphormolybänsaure Salze.}

Berzelius fand die Löslichkeit der Molybdünsäure in Phosphorsäure. L. Gmelin entdeckte das unlösliche gelbe Ammoniaksalz, Svanberg und Struve analysirten dasselbe, glaubten aber, gleich wie Gmelin, sein Gehalt an Phosphorsäure sei unwesentlich. Diesen Irrthum berichtigte Sonnenschein. Da das Salz seitdem vielfach zur Entdeckung und Bestimmung der Phosphorsäure dient, ist es auch 
oftmals analysirt worden, allein bis jetzt kennt man seine Zusammensetzung nicht mit Sicherheit, insofern nach den Versuchen von Sonnenschein, Seligsohn, Nutzinger, Spiess, Eggertz, Sopp and Debray $\mathrm{P}_{2} \mathrm{O}_{5}: \mathrm{MoO}_{3}=1: 20$ bis 1:29, $\mathrm{P}_{2} \mathrm{O}_{6}: \mathrm{Am}_{2} \mathrm{O}$ $=1: 2.4$ bis $1: 5$ ist. Am meisten scheint man der Angabe Debray's vertraut zu haben, der jene Verhältnisse hier und bei dem Kalisalz $=1: 20$ and $1: 3$ annimmt. Diesem Chemiker verdanken wir auch die Kenntniss der Phosphormolybdănsäure und verschiedener Salze, in denen beide Săuren in anderen Verhä'tnissen enthalten sind.

Veranlasst durch Versuche, über die Phosphorbestimmung im Roheisen u. s. w., habe ich versucht, zunächst die Zusammensetzung der unlöslichen, gelben Salze und der aus ihnen darch die Einwirknng von Alkalien entstehenden, farblosen und zum Theil krystallisirten Phosphomolybdate festzustellen.

Gelbes phosphormolybdänsaures Ammoniak.

Zur Fällung dienten eine salpetersaure Lösung von krystallisirtem, molybdänsaurem Ammoniak und freie Phosphorsäure. Das luftrockene Salz verliert über Schwefelsäure seinen ganzen Wassergehalt. Es ist alsdann bei $100^{\circ}$ beständig, entwickelt aber bei $120^{\circ}$ schon etwas Ammoniak. Als Mittel mebrerer gut stimmender Versuche erbjelt ich:

$$
\begin{array}{lr}
\text { Molybdänsäure . } & 86.45 \\
\text { Pbosphorsäure . } & 3.90 \\
\text { Ammoniumoxyd } & 3.25 \\
\text { Wasser . . . . . } & \frac{5.77}{100.37}
\end{array}
$$

Das Molekular - Verbältniss werden wir weiterhin erörtern.

Gelbes phosphormolybdänsaures Kalí.

Zu seiner Darstellung wurde Molybdänsäure mit kohlensaurem Kali geschmolzen, im Uebrigen wie vorher verfahren. Es gleicht im Ansehen ganz dem Ammoniaksalz, verliert aber das Wasser vollständig erst bei $120-140^{\circ}$; und erleidet dann selbst bei starkem Erbitzen keinen Verlust, so dass es für die Phosphorbestimmung dem Ammoniaksalz rorzuziehen ist. Unter den rerschiedenen analytischen Methoden finde ich diejenige am hesten, welche die Molybdän- und Phosphorsäure mittelst Quecksilberoxydulnitrat zunächst abscheidet.

Der Wassergebalt fand sich $=5.48-5.92 \mathrm{pCt}$.

Sieben Analysen gaben im Mittel:

$$
\begin{aligned}
& \text { Molybdänsäure } 84.43 \\
& \text { Phosphorsäure } \quad 3.78 \\
& \text { Kali . . . . . } 6.86(7.96)^{1} \text { ) } \\
& \text { Wasser } \cdots \frac{5.55}{100.62}
\end{aligned}
$$

1) Maximum. 
Vergleiclt man nun die aus den analytischen Mitteln beider Salze folgenden Molekular-Verhältnisse, so erhält man:

$\begin{array}{ccccc} & \mathrm{R}_{2} \mathrm{O} & \mathrm{P}_{2} \mathrm{O}_{5} & \mathrm{MoO}_{2} & \mathrm{H}_{2} \mathrm{O} \\ \text { Ammoniaksalz } & 2.98 & 1 & 21.9 & 11.7 \\ \text { Kalisalz } \ldots . .2 & 2.8 & 1 & 22.2 & 11.7 \\ \quad(3.1) & & & \\ \text { Also offenbar } & 3 & 1 & 22 & 12 \\ \mathbf{3} \mathrm{R}_{2} \mathrm{O}+\mathrm{P}_{2} \mathrm{O}_{3}+22 \mathrm{MoO}_{3}+12 \mathrm{aq} .\end{array}$

Durch Discussion von neun vorliegenden Analysen lässt sich zeigen, dass weder 20 noch $24 \mathrm{Mol}$. $\mathrm{MoO}_{3}$ vorhanden sein kōnnen, dass also Debray's Anoabme der ersten Zahl nicht richtig ist.

Zu demselben Resultat hat eine Reihe von Versuchen geführt, bei welchen eine Lösnng von phosphorsaurem Natron von genau bestimmtem Gehalt mit einer kalihaltigen, sauren Molybdäniösung gefüllt und der bei $140^{\circ}$ getrocknete Niederschlag gewogen wurde.

Verhalten der gelben Phosphomolybdate zu den Alkalien.

Längst bekannt ist die Löslichkeit des Ammoniaksalzes. Aus der Lösung in Ammoniak krystallisirt ein farbloses Salz, welches Zenker schon 1853 beschrieben und untersucht hat. Man erbält dasselbe noch Jeichter aus molybdänsaurem Ammoniak, Phosphosäure nnd überschüssigem Ammoniak. Die dünnen Prismenaggregate sind leicht löslich; ihre Analyse führte zu

$$
3 \mathrm{Am}_{2} \mathrm{O}+\mathrm{P}_{2} \mathrm{O}_{5}+5 \mathrm{MuO}_{3}+7 \mathrm{aq} \text {, }
$$

was mit Zenker's Resultaten genau übereinstimmt.

Durch Zusatz einer Sãure fällt aus der Lōsung sogleich wieder das gelbe Salx nieder, allein in dem Filtrat bleibt stets eine gewisse Menge Molybdänsäare, natürlich neben Pbosphorsãure and Ammoniak. Die ungleiche Menge der ersteren erlaubt weder unzweifelbaft daraus die Zneammensetzung des gelben Salzes abzuleiten, noch den Verlauf des Prozesses genau zu virfolgen.

\section{Verbalten des Kalisales zn Kali.}

Durch eine angemessene geringere Menge Kalilauge verwandelt sich das gelbe Salz in ein unlösliches, weisses, in einer grösseren Menge Kali löst es sich vollständig auf. Das weisse, unlōsliche Salz ist im getrockneten Zustande

$$
5 \mathrm{~K}_{2} \mathrm{O}+\mathrm{P}_{2} \mathrm{O}_{5}+15 \mathrm{MoO}_{3} \text {. }
$$

Durch Säuren wird es sogleich wieder in das gelbe Salz verwandelt, jedoch erbält man nicht immer dieselbe Menge von diesem; im Filtrat scheinen $\mathrm{K}_{2} \mathrm{O}, \mathrm{P}_{2} \mathrm{O}_{5}$ and $\mathrm{MoO}_{3}$ in dem Molekularverbältniss $6: 1: 12 \mathrm{zu}$ stehen.

Die Flüssigkeit, welche bei der Bildung dieses Salzes erhalten wird, seheidet beim Erhitzen eine grosse Menge dreifach molybdänsauren $\mathrm{Kalis} \mathrm{K}_{2} \mathrm{Mo}_{3} \mathrm{O}_{10}$ in käsigen Flocken ab. 
Das soeben beschriebene amorphe, weisse Salz löst sich mit Leichtigkeit in Kalilauge auf, und giebt beim Verdunsten Krystallrinden von normal 'm, molybdänsaurem Kali $\mathrm{K}_{2} \mathrm{MoO}_{4}$, welche ein wenig Phosphorsäure enthalten.

Debray giebt an, dass aus dem gelben $K_{a}$ ilisalz durch überschüssiges Kali ein dem Ammoniaksalz analoges mit 5 Mol. $\mathrm{MoO}_{3}$ enstehe. Es gelang mir anf diesem Wege nicht, die Verbindung rein zu exhaltén, 'wohl aber, indem ich $1 \mathrm{Mol} . \mathrm{K}_{2} \mathrm{CO}_{3}$ mit $2 \mathrm{MoO}_{3}$ schmolz und die Lösung mit Phosphorsäure versetzte. Nach dem Abdampfen bilden sich grosse, farblose, luftbeständige Krystalle, welche dem zweigliedrigen System angebören, Combinationen eines Rhombengktaeders und seines Dodekaids, mit dem Axenverbältniss $a: b: c=$ $0.72: 1: 0.715$, also nahezu viergliedrig sind; diese Krystalle verlieren beim Erhitzen Wasser und bestehen aus:

$$
\begin{array}{cc}
\text { Molybdänsäure } & 56.00 \\
\text { Phosphorsäure } & 11.60 \\
\text { Kali . . . . . . } & 21.09 \\
\text { Wasser . . . } & \frac{10.62}{99.31}
\end{array}
$$

Sie sind also die gesuchte Verbindung.

Durch Säuren wird die Lösung unter Abscheidung des gelben Salzes zersetzt.

Löst man dreifach molybdänsaures Kali in möglichst wenig Kalilauge auf, und fügt ein wenig Phosphorsäure hinzu, so schiessen aus der concentrirten Lösung lange, weisse, glänzende Prismen an, welche rechtwinklig und durch ein Rhombenoktaeder vierflächig zugespitzt sind. A us meinen Messungen folgt $a: b: c=0.983: 1: 0.821$. Sie verlieren ihren Wassergehalc bei $120^{\circ}$ und enthalten:

$$
\begin{array}{ll}
\text { Molybdänsäure } & 56.40 \\
\text { Phosphorsäure } & 11.33 \\
\text { Kali . . . . . . } & 17.97 \\
\text { Wasser } \ldots . . \frac{14.30}{100 .}
\end{array}
$$

Das Salz ist folglich

$$
5 \mathrm{~K}_{2} \mathrm{O}+2 \mathrm{P}_{2} \mathrm{O}_{5}+10 \mathrm{MoO}_{3}+20 \text { aq. }
$$

Es ist obne $Z_{w}$ eifel schon von $Z$ enker erhalten worden, jedoch hat er bei gleichem Kali- and Wassergehalt nur 7.24 pCt. Phosphorsäure gefunden.

Auch Debray führt die Salze

$$
3 \mathrm{R}_{2} \mathrm{O}+2 \mathrm{P}_{2} \mathrm{O}_{5}+10 \mathrm{MoO}_{3}
$$

auf, ohne sie jedoch zu charakterisiren.

Dass es von Säuren, unter Bildung des gelben Salzes zersetzt wird, bedarf kaum der Erwähnung; durch besondere Versuche fand ich, 


$\begin{array}{lccc} & \text { im Niederschlag } & \text { in Filtrat } \\ \mathrm{MoO}_{3} & 1 & : & 1.6 \\ \mathrm{P}_{2} \mathrm{O}_{5} & 1 & : & 11.3 \\ \mathrm{~K}_{2} \mathrm{O} & 1 & : & 8.1\end{array}$
liefern.

Es scheint also, als wenn 5 Mol. des Salzes 1 Mol. des gelben

\section{C. Rammelsberg: Ueber die Bestimmung des Kupferoxyduls im Kupfer. \\ (Vorgetragen in der Sitzung vom Verfasser.)}

Bei der Analyse von Garkupfer, sei es im Heerd oder im Flammofen (Raffinat) dargestellt, ist es von Wichtigkeit, einen Gehalt an Kupferoxydul zu bestimmen. Karsten ${ }^{1}$ ) hatte vorgescbrieben, das Kupfer mit einer Lösung von salpetersaurem Silber zu behandeln, welche das Oxydul unverändert lasse, so dass es in dem Gemenge mit Silber leicht zu bestimmen sei.

Schon vor langer Zeit hatte ich mich überzeugt, dass Karsten's Angabe ganz unrichtig sei, dass Kupferoxydul auf die Silberlösung kräftig einwirke, und ich hatte vorgeschlagen, den Gehalt an Kupferoxydul durch Glühen des Kupfers in Wasserstoffgas zu bestimmen ${ }^{2}$ ).

Neuerlich hat Hampe in einer Abhandlung über die Analyse des Kupfers diese Reduction ebenfalls empfohlen. Da sie aber die gesammte Sauerstoffmenge angiebt, von welcher ein Theil auf Rechnung anderer Oxyde (z. B. Nickeloxyd) kommen könne, so schreibt er vor, eine neue Probe mit Silberauflösung $\mathrm{zu}$ behandeln, um aus dem Kupfergehalt des reducirten Silbers das Oxydul zu berechnen.

Nun hat H. Rose vor zwanzig Jahren ${ }^{3}$ ) gefunden, dass bei der Einwirkung ron salpetersaurem Silber auf Kupferoxydul sicb neben Silber ein basiches Kupfernitrat fällt. Allein er hat weder die $\mathrm{Zu}$ sammensetzung dieses Salzes noch das Verbältniss des in Form von normalem Nitrat aufgelösten Kupfers zu dem im Rückstand befindlichen festgestellt. Es ist klar, dass man ohne Kenntniss dieses Verhältnisses nichts über die Menge des Kupferoxyduls wissen kann. Deshalb habe ich diesen Punkt durch einige Versuche aufzuklären gesucht.

Eine gewogene Menge Kupferoxydul wurde mit einem Ueberschuss von salpetersaurer Silberlösung digerirt. In dem blauen Filtrat fanden sicb, nacb Entfernung des Silbers $28.8 \mathrm{pCt}$. des angewandten Kupfers. Von der Fällung wurde ein Theil geglüht; er hinterliess

1) System der Metallurgie 5, 267.

2) S. meinen Leitfaden f. d. quant. Analyse. 2. Aufl., S. 233.

3) Pogg. Ann. 101, 513. 